\title{
Statistical Analysis of Accelerometer,Gyroscope with State Estimation
}

\author{
Ashvini Kulkarni, Augusta Sophy Beulet P.
}

\begin{abstract}
This paper describes the tracking of the object with the utility of a three-axis accelerometer and gyroscope for navigation. The sensor fusion is receiving enormous research interest which is used in monitoring and tracking position. For dynamic modeling, for real-time data, the perceptual mixing of the signals is required. For proper mixing of the signals, sensor fusion techniques are used. This paper presents the comparative review analysis for the three-axis accelerometer and gyroscope for linear acceleration and angular rotation.
\end{abstract}

Keywords: IMU, Kalman Filter, Sensor fusion

\section{INTRODUCTION}

$\mathrm{N}_{\mathrm{a}}$ avigation is the process of monitoring and controlling object position. This navigation is undergoing with various techniques and methods over these years, viz. compass, coastal navigation, dead reckoning, charts. Over these methods, the most popular method for navigation is preferred as GPS (Global Positioning System) nowadays. This is a preferred method to assist in knowing object location in computers and mobile phones. The problem with GPS is signal degradation particularly caused in the environment, loss of the signal because of the obstacles, trilateration to know the exact point of intersecting derived by the satellite signals and inaccurate signal due to the multipath [1].

Unfortunately GPS for navigation needs the clear sky to receive signals even though with more precise GPS signals in clear sky, signals are prone to errors. Thus some sort of filtration is required to provide navigation with certain conditions of the environment so that GPS receiver signals should not get affected by environmental conditions. It is required for GPS receiver signals to be noise-free, to get the exact position of the object. The presence of noise in received signals from GPS satellites may affect its position and it becomes difficult to maintain track of these objects in surveillance. Continual reception of noise-free signals is more important for navigation under such applications [2],[3]. To overcome the limitation of an inaccurate position of any object in GPS based navigation, DGPS (Differential Global Positioning) is promoted.

DGPS is mentioned to give location accuracy based on a ground-based reference station to calculate the difference between the actual position and the position received by GPS satellite [3] [4].
These base station roles are to broadcast the internal pseudo-range and actual pseudo-range and calculate the difference between two ranges and transmit through groundbased stations [5]. In order to support GPS the time based synchronization has to be taken for consideration of traffic with timely stability [6]. With respect to the time based synchronization, pseudo-range is one more factor for calculating the distance between the satellites. The navigation receivers among which determines the distances from them for calculating position along with Non-linear least squares method to identify position with the clock bias [7].To aid in navigation for the GPS signal to bear with erroneous signal, inertial navigation systems are taken into lead the navigation [8].

Vision-based approach is an approach for navigation which is gaining numerous interest in the field of navigation [9],[10].

In addition to these methods, another approach for navigation is MEMS (Micro Electro Mechanical System) based [11]. These sensors provide position through the accelerometer and gyroscope. These sensors acquire acceleration and the rotational movements of object position. Such signals from the sensors are prone to drift error, sampling error, etc. The three-axis accelerometers measure the inertial forces on the three axes. Similarly, each gyroscope measures the rotation in each axis. Accelerometer and gyroscope are the most widely used sensors for navigation. These sensors deliver velocity and angular rate information around the axis. Through this type of information the exact location of an object can be obtained. Visual monitoring and tracking, security, surveillance are some of the applications of navigation. There are various techniques implemented with high-end precision in navigation with state estimation recursive algorithm. This paper presents the analysis of the various methods for detecting acceleration and angular position of an object with a different approach for knowing its position in navigation applications.

The paper is organized as follows. Section II describes the basic formulation of a three-axis accelerometer and its implementation and describes three-axis gyroscopes for measurement of rotation around axes. Section III consists of accelerometer and gyroscope testing on the Kalman filter.

All the results are discussed in section IV followed by a conclusion and future work is discussed in section $\mathrm{V}$.

\footnotetext{
Revised Manuscript Received on December 05, 2019

Ashvini Kulkarni, School of Electronics Engineering, Vellore Institute of Technology, Chennai, India Email: ashvini.kulkarni11@gmail.com

Augusta Sophy Bulet P, School of Electronics Engineering, Vellore Institute of Technology, Chennai, India Email: augustasophyt.p@vit.ac.in
} 


\section{ACCELERATION FORCE AND ROTATIONAL MOTION}

\section{A.3-axis Accelerometer}

Accelerometers are responsible to measure the accelerometer forces. These are usually detected force that is directed in the opposite direction from the acceleration vectors often called a fictitious force [12]. These accelerometer measures all linear forces. There are two types of accelerometers viz. AC response accelerometer and DC response accelerometer. AC response category can measure only dynamic events as AC coupled systems [13]. They are not suitable to measure static responses like gravity and centrifugal forces whereas DC response accelerometers can measure static as well as dynamic events for an object. The commonly used accelerometer is in the $\mathrm{AC}$ response category. Piezoelectric elements are used in these types of accelerometers and for the DC response there are two basic elements capacitive and piezo-resistive. Capacitive categories of the accelerometer are more popular and it is fabricated using MEMS-based technology. These are the types of accelerometer that measures acceleration on the surfaces using capacitive method which has capability to measure static and dynamic accelerometer response.

The accelerometer measures the acceleration in the three directions which is always affected by the gravitational force [14].

$a_{d}=-g-\sum \frac{F}{m}$

Where,

$m=$ mass of the body

$g=$ force of gravity

$a_{d}=$ acceleration applied to the body

As we assume the linearity, responsivity is expressed as a vector form:

$\vec{r}=r \hat{r}=r_{x} \widehat{x}+r_{x} \widehat{y}+r_{x} \widehat{z}$

This equation is expressed as a vector in $\{\mathrm{x}, \mathrm{y}, \mathrm{z}\}$ coordinate systems.

Where

$r=\left\|\rightarrow{ }_{r} \quad\right\|$ Magnitude vector of $\mathrm{r}$

$\hat{r}=$ maximum responsivity in coordinate systems $\{\mathrm{x}, \mathrm{y}, \mathrm{z}\}$

For accelerometer response vector is expressed as

$\overrightarrow{R_{r}}=R_{r x} \widehat{x}+R_{r y} \hat{y}+R_{r z} \widehat{z}$

Acceleration vector for the same coordinate systems denoted as:

$\vec{a}=a_{x} \widehat{x}+a_{x} \widehat{y}+a_{x} \widehat{z}$

Most of the accelerometers are of two types, analog and digital. The accelerometer will output voltage levels within a predefined range that need to be converted into digital values. This conversion is done by using an ADC. For the conversion of the input reference voltage is required as well as bit conversion for ADC is needed. This output voltage is expressed as:

voltsRx $=\frac{\text { ADCR } * \text { Vref }}{\left(2^{\text {ADC no.of bits }}\right)-1}$

(5)

Each accelerometer has zero voltage levels. To get the signed voltage values we need to calculate the shift from these levels which is represented by VzeroG.

$\Delta$ voltsRx $=A D C R x-$ VzeroG

\section{$\Delta$ voltsRy $=A D C R y-$ VzeroG \\ (7) \\ $\Delta$ voltsRz $=A D C R z-$ VzeroG}

(8)

Above three equation gives accelerometer reading for three axes in volts to express same in $g$ it is mandatory to apply sensitivity which is expressed as

$$
\begin{aligned}
& R x=\frac{\Delta \text { voltsRx }}{\text { Sensitivity }} \\
& R y=\frac{\Delta \text { DoltsRy }}{\text { Sensitivity }}
\end{aligned}
$$

(10)

$R z=\frac{\Delta \text { voltsRz }}{\text { Sensitivity }}$

The above three equations give accelerometer reading for inertial vector.

\section{B. 3-axis gyroscope}

Gyroscope is used to measure rotational motions. Usually, gyroscope used to provide direction in the navigation systems. Gyroscope maintains its performance for simply to be able to measure the rate of rotation around with some fixed particular axis. These types of sensors are used to regulate an angular position based on the basis of principle of the rigidity in space [15]

Gyroscope gives the output which is linearly related to the rate of change of the angle' $s$ ' of projection of the vectors on that plane. Consider the z-axis and the $R x y$ and $R y z$ vectors on that plane and let's assume the measured angle of rotation around $\mathrm{Y}$-axis at time t0 and measured the same angle after some time $\mathrm{t} 1$, so the angle between the Axz plane with $\mathrm{z}$-axis is given by:

RateAxz $=\frac{\left(A_{x z 1}-A_{x z 0}\right)}{(\mathrm{t} 1-\mathrm{t} 0)}$

This value needs to be converted as given as

$\begin{aligned} \triangle A X Z & =\frac{\frac{\text { AdcGyroxz } * \text { ADCVref }}{\text { 1023-VzeroRate }}}{\text { Sensitivity }} \\ \triangle A y Z & =\frac{\frac{\text { AdcGyroyz } * \text { ADCVref }}{1023-\text { VzeroRate }}}{\text { Sensitivity }}\end{aligned}$

\section{EFFECT OF KALMAN FILTER ON ACCELEROMETER AND GYROSCOPE}

For the prediction and estimation, the Kalman filter gives perspicacity in the prediction and estimation of the objects. This filter is subjected to determine state-space estimation. In Kalman filter dynamic linear model, the initial object position has to undergo with prediction stage which adds measurement noise and observation noise over time. Hence the corrected position is accomplished with the state estimation, measurement and update stages. This dynamic system can be distributed with noise. The data from the sensor has to be given to the Kalman filter [16].

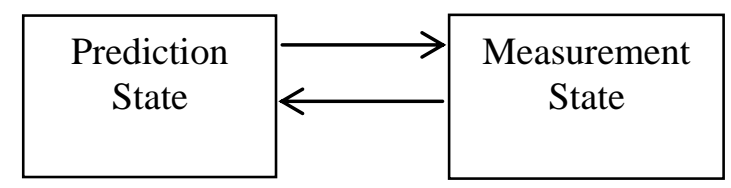

Fig. 1: Kalman filter conceptual model

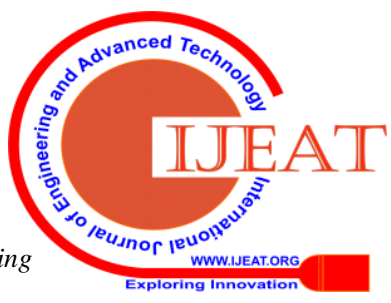


Kalman states the basic two equations to be followed for the measurement and prediction of the systems [17],[18]. $x_{k+1}=A x_{k}+B u_{k}+w_{k}$

Where

$x=$ System state

$\mathrm{u}=$ Input to the system

$k=$ Time index

$\mathrm{w}=$ Process noise

$A, B=$ Matrix of the system

Kalman filter gives the output with added noise as:

$y_{k}=C x_{k}+z_{k}$

Where

$\mathrm{z}=$ Noise measurement

$\mathrm{y}=$ Output measured

Above equations are the vectors that contains information for example, $x$ vector has all the information regarding the present state. The output of the filter contains the information measured with time and if the object is traveling in a straight line then the object has velocity $v$ and object position $p$. And this acceleration is getting changed at time $T$ so the velocity is given by,

$v_{k+1}=v_{k}+T u_{k}$

Usually, the updated velocity is represented by the current velocity with prescribed velocity within the specified time. Here the chances of noise as the previous velocity might not be true due to some random noise in the velocity. So (17) can be rewritten as:

$v_{k+1}=v_{k}+T u_{k}+v_{k n}$

Where

$v_{k n}=$ Velocity noise

Similarly, the position for an object is calculated as:

$P_{k+1}=p_{k}+T v_{k}+\frac{1}{2} T^{2} u_{k}+p_{k n}$

Where

$v_{k n}=$ Position noise

Now to represent the velocity and position we can define these vectors that has velocity and position so the system equation is represented as

$x_{k+1}=\left[\begin{array}{ll}1 & T \\ 0 & 1\end{array}\right] x_{k}+\frac{1}{2} T^{2} u_{k+} w_{k}$

$y_{k}=\left[\begin{array}{ll}1 & 0\end{array}\right] x_{k}+z_{k}$

Where

$z_{k}=$ Process noise

Kalman filter always comprises of measurement noise and process noise. So there is a correlation of process noise and measurement noise, in terms of the noise covariance matrices covariance noise is given as:

$S_{w}=E\left(w_{k} w_{k}^{T}\right)$

And measurement noise covariance is given by:

$S_{z}=E\left(z_{k} z_{k}^{T}\right)$

This Kalman filter has two important stages of prediction and correction stages with all in presence of Gaussian noise and given by following equations [19],[20]:

$$
\begin{aligned}
& K(k)=C^{+}(k) H^{T}(k)\left[H(k) C^{+}(k) H^{T}(k)+R(k)\right]^{-1} \\
& (23) \\
& x^{\wedge}(k)=x^{+}(k)+K(k)\left[z(k)-H(k) x^{+}(k)\right] \\
& C^{\wedge}(k)=[I-K(k) H(k)] C^{+}(k) \\
& (25) \\
& x^{+}(k+1)=\Phi(k) x^{\wedge}(k) \\
& C^{+}(k+1)=\Phi(k) C^{\wedge}(k) \Phi^{T}(k)+Q(k)
\end{aligned}
$$

Where

$\mathrm{K}=$ Kalman gain

$\mathrm{x}^{\wedge}=$ State estimate

$\mathrm{C}^{\wedge}=$ Covariance estimate of state

$\Phi=$ State transition matrix for propagation of the state forward in time from discrete-time $\mathrm{k}$ to $\mathrm{k}+1$.

$\mathrm{H}=$ Measurement matrix that relates measurements to the state

$\mathrm{R}=$ Priori measurement noise covariance matrix is the prior process noise covariance matrix

$\mathrm{Z}=$ Measurement vector. Prediction of state and

Covariance estimates one step forward in time indicated by + sign.

The position of the object about the sensor values is described by its coordinates i.e. accelerometer values and gyroscope values prescribed by the sensor and the position of object varies over time by equation no.27.

\section{TEST AND RESULTS}

To test the sensor-based approached for the individual sensor approach is based on the raw sensor data which is received by sensor MPU6050. This is a three-axis accelerometer and three-axis gyroscope MEMS-based sensor. The experimental route area is conducted in an indoor environment. The accelerometer and gyroscope raw date are taken and analyzed based on its linear acceleration performance on the three axes. The same analysis is attempted for the three-axis gyroscope. Accelerometer corresponds to the $0 \mathrm{~m} / \mathrm{s}^{2}$ and voltage levels which measures the voltage shifts i.e. $1.65 \mathrm{~V}$ and as the output of the accelerometer is in volts and to serve the purpose to convert into sensitivity expressed $\mathrm{mV} / \mathrm{g}$ requires the sensitivity and as per MPU6050, it is taken as sensitivity scale factor 16384 with accelerometer full-scale range \pm 2 [21]. Fig. 2 shows the raw linear acceleration data for accelerometer in MPU6050. The analysis of the raw data is conducted in MATLAB.

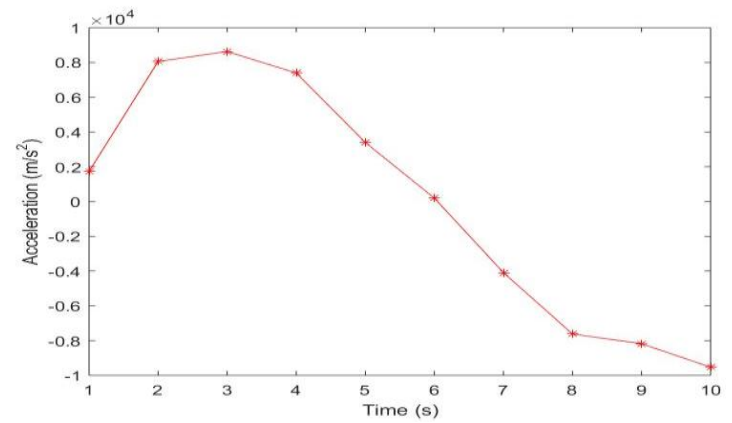

Fig. 2: Three-axis accelerometer raw data from the sensor

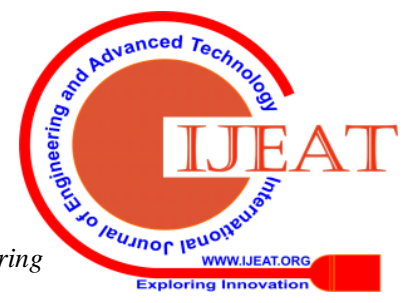




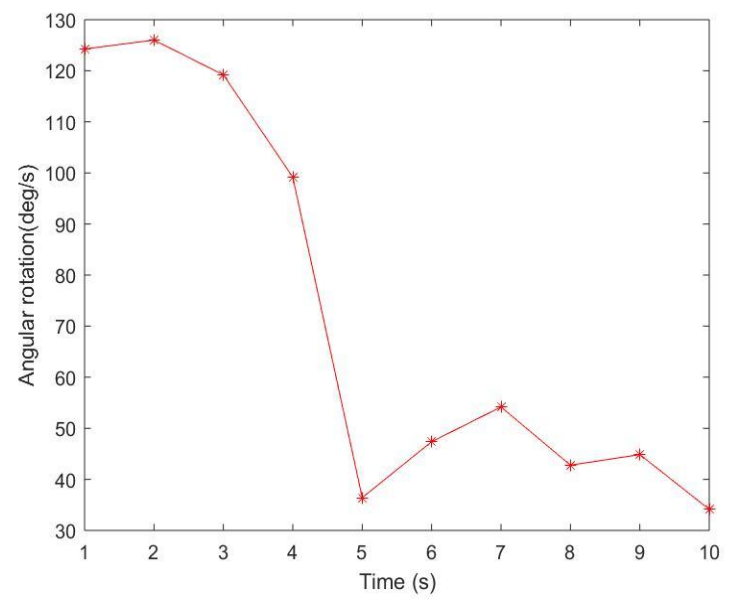

Fig. 3: Gyroscope around the axes from sensors with sensitivity is considered as $1.31 \mathrm{mV} / \mathrm{g}$

Another method tested here is to get the acceleration and the angular movement by using the Kalman Filter. This filter works based on the prediction and the estimation and based on that it updates the measurement state. The same concept is applied to the accelerometer and gyroscope data. This raw data is the input to this filter and based on that it measures the estimation state. In estimation there is possibility of the measurement noise and the process noise to get added. We have assumed 0.6 as a measurement noise and process noise as 1 .

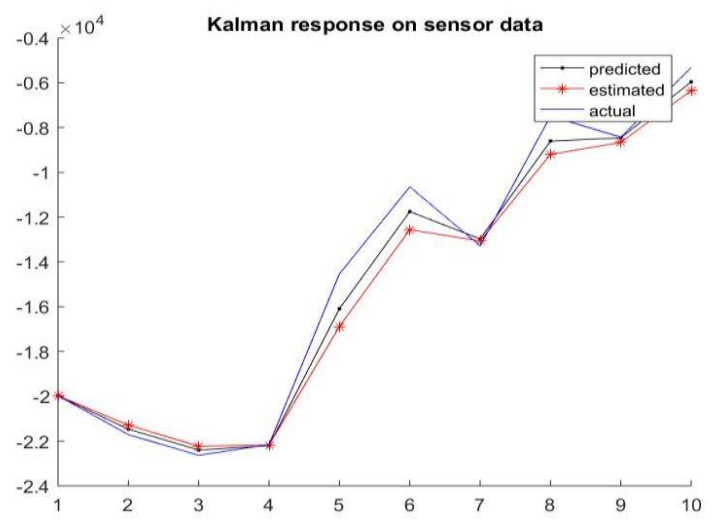

Fig. 4: Effect of Kalman filter on the raw accelerometer data

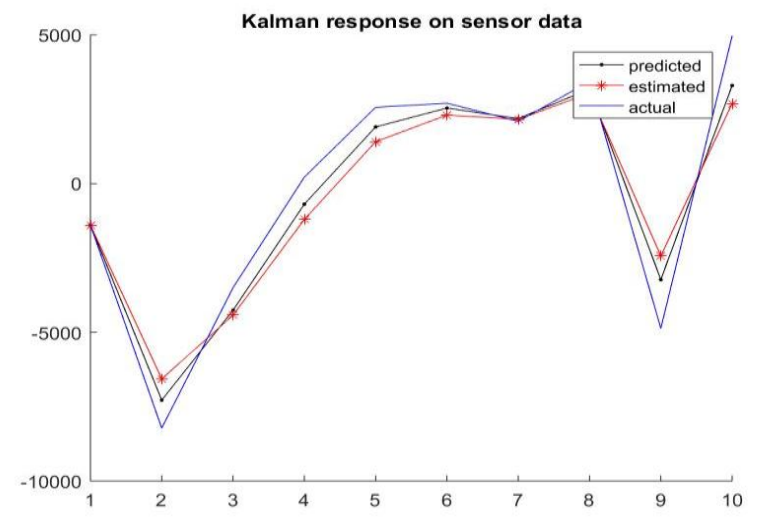

Fig. 5: Effect of Kalman filter on the raw accelerometer data

Above fig. 4 and fig. 5 shows the Kalman filter performance on the sensor data. This plot also gives the result with predicted values and the estimated values are the same with the actual sensor data.

\section{CONCLUSION AND FUTURE WORK}

As acceptance of the navigation, popularity is gained by the GPS but to some extent, these GPS signals have an erroneous signal which cannot give an accurate position. Similar to the navigation approaches the sensor itself is giving raw data and this data can be utilized to find out velocity and angular moments for the position. These sensors functionality can be used for navigation assistance which will track one's position. These sensory data also experience some noise that affects the raw data coming from sensors to find out the position from sensor raw data we approach of linear acceleration is implemented and shown in fig. 2 and fig. 3 although the accelerometer is affected by vibration and noise. For gyroscope, it is less sensitive to linear movement but gyroscope doesn't get to zero value when it stops because of drift problems in it. The results are better with the two different data for finding one's position.

In the further part, the same data is tested using Kalman filter and the analysis shows a better estimation for the data using the filter. This filter gives the satisfactory projection of the estimated data values and the predicted data values and the analysis of them is nearing to the actual data samples in it. Accelerometer and gyroscope have different sampling rate and the output of the sensors have their advantages but by combining both the data together can give better results in the navigation as a part of data fusion. Developing a methodology to effectively combine the different sensors data, their synthesis and analysis would be the future work.

\section{REFERENCES}

1. Michael Wright, Dion Stallings, and Dr. Derrek PU, "The Effectiveness of Global Positioning System Electronic Navigation " IEEE Southeast Con, 2003. Proceedings. ISBN: 0-7803-7856-3 , 03 March 2004

2. Kia Fallahi, Chi-Tsun Cheng, Michel Fattouche, "Robust Positioning Systems in the Presence of Outliers Under Weak GPS Signal Conditions", IEEE Systems Journal ( Volume: 6, Issue: 3, Sept. 2012 ), Page(s): 401 - 413 ISSN: 1937-9234

3. Gianfranco Fornaro,Nicola D'Agostino,Roberta Giuliani,Carlo Noviello,Diego Reale,Simona Verde," Assimilation of GPS-Derived Atmospheric Propagation Delay in DInSAR Data Processing", IEEE Journal of Selected Topics in Applied Earth Observations and Remote Sensing,Volume: 8, Issue: 2, Feb. 2015, Page(s): $784-799$,ISSN: 1939-1404

4. Susan Skone and Sudhir M. Shrestha, "Limitations in DGPS positioning accuracies at low latitudes during solar maximum" Geophysical Research Letter, published 28 May 2002

5. Kar-Ming Cheung, Charles Lee," A Trilateration Scheme for Relative Positioning", IEEE Aerospace Conference ISBN: 978-1-5090-1613-6, March 2017

6. Peter A Crossley, Hao Guo, Zhao Ma, "Time Synchronization for Transmission Substations Using GPS and IEEE 1588", Journal of Power and Energy Systems, VOL. 2, NO. 3, Sepetember2016, Page(s): 91 - 99,ISSN: 2096-0042

7. Xiaojing Du, Li Liu, Huaijian Li, "Experimental Study on GPS Nonlinear Least Squares Positioning Algorithm", International Conference on Intelligent Computation Technology and Automation, 11-12 May 2010, Page(s): 262 - 265, ISBN:978-1-4244-7280-2

8. Maiying Zhong, Jia Guo, Zhaohua Yang, "On the real-time performance evaluation of the inertial sensors for INS/GPS integrated systems", IEEE Sensors Journal ( Volume: 16, Issue: 17, Sept.1, 2016), Page(s): 6652 - 6661, ISSN: 1558-1748

9. Least Squares Positioning Algorithm", International Conference on Intelligent Computation Technology and Automation, 11-12 May 2010, Page(s): 262 - 265, ISBN:978-1-4244-7280-2

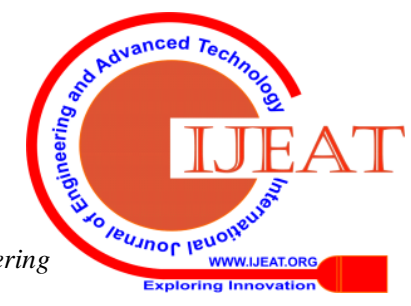


10. Jos’e Gaspar, Jos'e Santos-Victor, "Vision-based Navigation and Environmental Representations with an Omni-directional Camera", 2015 IEEE International Conference on Big Data 978-1-4673-72787/15, DOI 10.1109/BigDataCongress.2015.103

11. Zhou Haoyin, Zhana Tao, "A Vision-Based Navigation Approach with Multiple Radial Shape Marks for Indoor Aircraft Locating", Chinese Journal of Aeronautics, doi.org/10.1016/j.cja.2013.12.005,Volume 27, Issue 1, February 2014, Pages 76-84

12. Zheming Wu, Zhenguo Sun, Wenzeng Zhang, Qiang Chen, "A Novel Approach for Attitude Estimation Based on MEMS Inertial Sensors using Nonlinear Complementary Filters", IEEE Sensors Journal ( Volume: 16, Issue: 10, May 15, 2016 ), Page(s): 3856 - 3864, ISSN: $1558-1748$

13. D. Chattaraj*, K.B.M. Swamy and S. Sen, "Design and Analysis of Dual Axis MEMS Accelerometer", International Conference on Electronics and Communication Systems (ICECS), DOI 0.1109/ECS.2015.7125014, 26-27 Feb. 2015

14. Zdzisław Kowalczuk, Tomasz Merta, "Evaluation of position estimation based on accelerometer data", International Workshop on Robot Motion and Control, Poznan University of Technology, Poznan, Poland, ISBN: 978-1-4799-7043-8, July 6-2015

15. O. J. Woodman, "An introduction to inertial navigation," University of Cambridge, Computer Laboratory, Cambridge, UK, Tech. Rep. 696,2007

16. J.Z.Sasiadek, P. Haryana, "GPS/INS sensor fusion for accurate positioning \& navigation based on Kalman filter", IFAC Proceedings Volumes, Volume 37, Issue 5, June 2004, Pages 115-120

17. Mohinder S.Grewal, Angus P.Andrew, "Kalman Filtering: Theory and Practice Using MATLAB", Second Edition, Published by John Wiley \& sons, 2001

18. Sen Qiu, Zhelong Wang, Hongyu Zhao, Kairong Qin, Zhenglin Li, Huosheng $\mathrm{Hu}$, "Inertial/Magnetic Sensor Based Pedestrian Dead Reckoning by Means of Multi-Sensor Fusion" Information Fusion, Volume 39, Page No.108-119, Science direct, April 2017

19. Hang Geng, Yan Liang, Yurong Liu, Fuad E. Alsaadi, "Bias estimation for asynchronous multi-rate multi-sensor fusion with unknown inputs" Information Fusion, Volume 39, Page No.139-153, Science direct, April 2017

20. Kajiro Watanabe, Kazuyuki Kobayashi, and Fumio Munekata, "Multiple Sensor Fusion for Navigation Systems", Vehicle Navigation information System IEEE Conference proceedings, 06 August 2002, DOI:10.1109/ VNIS.1994.396787

21. InvenSense, "MPU-6000and MPU-6050 Product Specification," InvenSense Inc., Document Number: PS-MPU-6000A-00Revision: 3.4Release Date: 08/19/2013

\section{AUTHORS PROFILES}

Ms. Ashvini Kulkarni completed her Bachelor of Engineering in the stream of Electronics Engineang from D.Y. Patil College of Engineering, Kolhapur,Maharashtra and her Masters in the field of Communication Engineering from Marathwada Institute of Technology, Aurangabad, Maharashtra Presently she is working as Assistant Professor with International Institute of Information Technology, Pune, Maharashtra in Electronics \&
Department.She is pursuing his Ph.D at Vellore

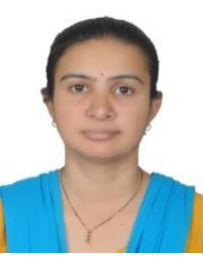
Telecommunication Department.She is pursuing his Ph.D at Vellore
Institute of Technology, Chennai and her area of interests includes Computer Vision, Embedded systems, IoT and Artificial Intelligence

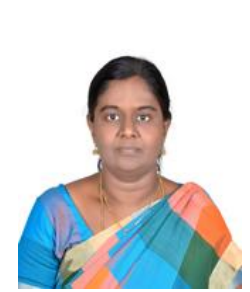

Dr. Augusta Sophy Bulet P. being a lecturer for more than 25 years, Dr.P.Augusta Sophy, has developed a passion towards teaching Engineering subjects and guiding projects. The passion has naturally been extended towards developing the students too. Have handled more than 20 differen subjects in Electronics and Communication Engineering and guided more than 50 projects in UG and PG levels. She has published 24 research papers in International Conferences and Journals.

She did her research work in the field of VLSI Signal Processing in Anna University, in 2015. Received her M.E degree from Anna University and finished her B.E degree in Government College of Engineering, Tirunelveli. Now she is working as Associate Professor in VIT University, Chennai. 